\title{
Epidemiology of Pregnancy-Associated ICU Utilization in Texas: 2001 - 2010
}

\author{
Lavi Oud
}

\begin{abstract}
Background: ICU admission is uncommon among obstetric patients. Nevertheless, the epidemiology of ICU utilization is considered to be a useful proxy for study of severe maternal morbidity and near-miss events. However, there is paucity of population-level studies in obstetric patients in the United States.

Methods: The Texas Inpatient Public Use Data File and state-based reports were used to identify pregnancy-associated hospitalizations and those involving admission to ICU $(\mathrm{n}=158,410)$ for the years 2001 - 2010. The clinical characteristics, outcomes, and the overall incidence and temporal trends of ICU admission were examined and stratified analyses of pregnancy outcomes were performed in specific categories of pregnancy-associated hospitalizations. In addition, ICU utilization among hospitalizations with maternal complications and organ dysfunction was evaluated.
\end{abstract}

Results: Chronic comorbidities (9.7\%) and presence of organ dysfunction $(6.2 \%)$ were uncommon among ICU admissions, with $26.5 \%$ having high severity of illness. The incidence of ICU admission was 39.0 per 1,000 pregnancy-associated hospitalizations-years. Marked variability was found in ICU admission both across pregnancy outcomes (ranging from 0.6 per 1,000 abortions-years to 85.9 per 1,000 stillbirths-years) and categories of pregnancy-associated hospitalizations (ranging from 32.1 per 1,000 delivery hospitalizations-years to 144.8 per 1,000 postpartum hospitalizations-years). The incidence of ICU admission rose $68 \%$ among pregnancy-associated hospitalizations and for all examined subgroups, except abortion. Preeclampsia/ eclampsia $(23.3 \%)$ and obstetric hemorrhage $(6.9 \%)$ were the most common maternal complications among ICU admissions. Four hundred fourteen women $(0.3 \%)$ died, while $97.6 \%$ were discharged home.

Conclusions: This study documents the highest incidence of ICU utilization in obstetric patients in the US to date. The findings sug-

Manuscript accepted for publication December 16, 2016

Division of Pulmonary and Critical Care Medicine, Department of Internal Medicine, Texas Tech University Health Sciences Center at the Permian Basin, 701 W. 5th St., Odessa, TX 79763, USA.Email: lavi.oud@ttuhsc.edu

doi: https://doi.org/10.14740/jocmr2854w gest low threshold for obstetric ICU admissions in the state and do not support comparative use of ICU utilization as surrogate measure for populations' burden of severe maternal morbidity and near-miss events. Nevertheless, the demonstrated tremendous heterogeneity in ICU utilization across examined subgroups identifies new high-risk groups of obstetric patients that would benefit from heightened clinician vigilance and timely ICU triage and care. Further studies are needed to inform reduction in avoidable variability in ICU utilization to both enhance maternal, fetal, and neonatal outcomes and to improve resource allocation.

Keywords: Intensive care unit; Pregnancy; Resource utilization; Mortality

\section{Introduction}

The obstetric population in the United States (US) includes increasingly older women [1] and those with chronic comorbidities [2]. When coupled with concomitantly changing obstetric practice, including rising use of cesarean section $[1,3]$, these changes are thought to be key sources of the observed rising rates of maternal morbidity during hospitalization [4].

Because maternal mortality rates became relatively rare [5], there has been increasing focus on addressing severe maternal morbidity and near-miss events $[4,6]$ as targets for improving maternal outcomes and as performance measure. Thus, studies of the epidemiology of ICU admissions of obstetric patients are considered to represent a useful proxy of severe maternal morbidity and near-miss events that can inform interventions to improve maternal outcomes [6-8]. However, there has been no agreed upon definition of severe maternal morbidity [6] and the recent consensus-based criteria for near-miss events put forward by the World Health Organization (WHO) [9] remain controversial [10, 11]. In addition, while contemporary guidelines stress the overarching goal that ICU admissions should be reserved for those patients who are likely to benefit [12], there have been no evidence-based data to guide clinicians in triaging obstetric patients to ICU $[13,14]$.

Although numerous studies examined obstetric critical care utilization $[6,8]$, the majority were single-centered, often involving tertiary centers [6], limiting the interpretation and 
generalizability of their findings. Only few population-level studies were reported to date on the epidemiology of obstetric ICU utilization in high-resource countries $[7,10,15,16]$. However, there is marked variability in international availability of critical care resources and practice patterns [17]. Thus, international population-based findings cannot be readily applied to specific countries.

Because national data sets in the US do not include data on ICU admission, population-based studies have been restricted to individual states. Previous state-level investigations of ICU utilization in obstetric populations in the US showed nearly four-fold difference between states $[7,16]$. The sources of the marked variability and its applicability to the US population at large remain unknown.

The increasing rates of maternal morbidity among hospitalized women can be expected to affect ICU utilization over time. However, only one study to date has examined temporal trends of ICU utilization, showing no change between 1999 and 2008 in Maryland [7].

Another limitation of contemporary reports on the epidemiology of obstetric ICU utilization involves the methodology used to derive the incidence of ICU admission. Investigators generally used the number of delivery hospitalizations as denominator [6]. The likely reason for this approach has been the comparative ease of access to delivery data in the hospital setting. In addition, use of a single denominator can facilitate comparison between studies.

However, using exclusively delivery events to examine the epidemiology of ICU utilization in obstetric patients entails considerable limitations. Data to date could not quantify the overall demand for ICU services in non-delivery patient strata (i.e., women having fetal loss or induced abortion) in the obstetric population at large, nor for the whole obstetric population. In addition, investigators generally did not address ICU utilization in non-delivery hospitalizations (i.e., anterpartum, postpartum) $[15,16]$. Non-delivery hospitalizations involve for the most part women with complications brought by or aggravated by pregnancy, who thus would be expected to have higher rates of ICU utilization, as compared to delivery hospitalizations, where the majority of women have relatively uneventful clinical course. When anterpartum or postpartum hospitalizations are examined separately, the use of delivery hospitalizations as denominator can lead to substantial underestimation of their demand for critical care services, as both groups comprise only a minority of all pregnancy-associated hospitalizations, reported to account for $8.8 \%$ of the whole cohort in a recent study [7]. That study showed that the incidence of ICU admission was markedly lower among non-delivery hospitalizations when indexed to delivery hospitalizations [7].

Finally, data on ICU utilization within specific categories of maternal morbidities can refine future study of interventions to limit their adverse effects. However, population-based studies to date generally did not examine these data, with the exception of a single report, addressing this area indirectly [7].

The objectives of the present study were to better characterize the epidemiology of ICU utilization in the obstetric population at large and among pregnancy-associated hospitalizations in order to inform future investigations on optimal resource allocation and preventive and interventional tools to improve maternal outcomes at a clinician- and health care system levels. To do so, we analyzed data from the Texas Inpatient Public Use Data File (TIPUDF), which tracks information on non-federal inpatient hospitalizations and includes data on ICU utilization.

\section{Materials and Methods}

This was a retrospective population-based cohort study. Because we used a publicly available, de-identified data set, this study was determined to be exempt from formal review by the Texas Tech Health Sciences Center Institutional Review Board.

\section{Setting and data sources}

We used the TIPUDF to examine ICU utilization by obstetric patients in the state. TIPUDF is an administrative data set maintained by the Texas Department of State Health Services [18]. The use of TIPUDF has been previously described [19]. Briefly, the data set includes de-identified inpatient discharge data on the demographic, clinical, resource utilization, and outcome domains from state-licensed, non-federal hospitals, and captures $93-97 \%$ of all hospital discharges in the state. The data set does not provide linkage of pregnancy-associated hospitalizations to corresponding fetal outcomes (other than fetal loss or stillbirth) or to neonatal outcomes.

Data on the annual number of live births, abortions, and fetal deaths (stillbirths) [20] were obtained from the Vital Statistics Annual Reports, compiled by the Center for Health Statistics at the Texas Department of State Health Services [21].

\section{Study population}

We used International Classification of Diseases, Ninth Revision, Clinical Modification (ICD-9-CM) codes (Supplementary Table 1, www.jocmr.org) to identify Texas residents with pregnancy-associated hospitalizations between 2001 and 2010. We categorized the type of pregnancy-associated hospitalizations into the following mutually exclusive, hierarchical groups, using pregnancy-associated ICD-9-CM codes (Supplementary Table 1, www.jocmr.org): 1) fetal loss (pregnancies with abortive outcome, excluding induced abortion); 2) induced abortion (termed abortion in the remainder of manuscript); 3) delivery (based on the approach described by Kuklina et al [22]); 4) postpartum (hospitalizations with an ICD-9-CM code for puerperal complications, without pregnancy-related diagnosis codes of groups 1-3), and 5) antepartum (hospitalization with pregnancy-related diagnosis, but without pregnancy-related diagnosis codes of groups 1-4). In addition, we examined pregnancy-associated hospitalizations with stillbirths (ICD-9-CM codes V27.1, V27.3, V27.4, V27.6, V27.7, 656.4) as a separate category from the aforementioned mutually exclusive groups because stillbirth events can be coded as otherwise antepartum 
and delivery hospitalizations.

\section{ICU admissions}

Hospitalizations with ICU admission were identified based on unit-specific revenue code for an ICU or a coronary care unit. Intermediate care units or step-down units were not included. Because administrative data sets do not include information of the temporal course of hospitalization, it could not be determined whether ICU admissions occurred at the start of hospitalization or later during hospital course. Similarly, the specific indications for ICU admission and whether ICU admission preceded or followed fetal loss, stillbirth or delivery cannot be determined from the administrative data.

\section{Data collection}

We collected data on patients' age, race/ethnicity (categorized as non-Hispanic black (black), non-Hispanic white (white), Hispanic, and other), health insurance (categorized as private, Medicaid, uninsured, and other), and comorbid conditions (based on the Deyo modification of the Charlson comorbidity index [23]). Because there is no agreed upon definition of severe maternal morbidity, we used the categories of causes of maternal death tracked by the CDC [5] and additional maternal morbidities reported by others [7] to identify maternal complications (Supplementary Table 2, www.jocmr.org). In addition, we collected data on the number and type of organ dysfunction, as previously defined [24] (Supplementary Table 3 , www.jocmr.org), as proxy for near-miss events. Because administrative data sets do not include physiological information used to derive severity of illness scores for ICU patients, we planned to examine the number of organ dysfunctions as surrogate measure. However, because organ dysfunction was reported only in a small minority of pregnancy-associated ICU admissions, we used the All Patients Refined Diagnosis Related Groups (APR-DRG) severity of illness indicators, routinely reported in administrative data and comprised of four categories including "minor", "moderate", "high", and "extreme". The severity of illness indicators are based on proprietary grouping software developed by $3 \mathrm{M}$ Health Information Systems and integrates patient's demographics, principal and secondary diagnoses, and procedure codes. Thus, patients with increasing number of comorbidities and complications (i.e., organ dysfunction) would have higher severity of illness.

\section{Outcomes}

The primary outcome was the incidence of ICU admission among: 1) all pregnant women in the state of Texas and within specific categories of pregnancy outcomes (feta loss, abortion, live births, and stillbirths); and 2) all pregnancy-associated hospitalizations and within specific categories of hospitalization (fetal loss, induced abortion, antepartum, delivery, and postpartum). The secondary outcome was the rate of ICU ad- mission within each category of maternal complications and organ dysfunction.

\section{Data analysis}

Because TIPUDF provides discharge-level, rather than patient-level information and thus precludes proper accounting for readmissions in the data set, we report number of hospitalizations rather than number of patients.

In order to derive an estimate of the total number of pregnancies in Texas across the full spectrum of pregnancy population at risk, we calculated the number of total estimated pregnancies (TEP). TEP for a given year was a combination of the number of live births, fetal deaths (stillbirths) (events reported by the state, occurring at $\geq 20$ weeks of gestation), abortions, and estimates of the annual number of fetal losses (events occurring at $<20$ weeks of gestation, including miscarriage, ectopic and molar pregnancies). The process to estimate the annual number of fetal losses was previously reported [25]. There were 1,783 hospitalizations $(0.04 \%)$ out of all pregnancy-associated hospitalizations and 1,266 (0.8\%) of those with ICU admission associated with fetal loss/abortion that could not be adequately classified to only one group (that is, either fetal loss or abortion), because their only pregnancyassociated ICD-9-CM code was 639.XX (complications following abortion and ectopic and molar pregnancies). We thus excluded these hospitalizations from the descriptive categories of pregnancy-associated hospitalizations among ICU admissions and from subgroup analysis of the incidence of specific subgroups outlined under outcomes. To assure consistency, we used the term fetal loss throughout the manuscript to denote the terms spontaneous abortion or miscarriage used in other reports.

Because the demand for ICU services among non-delivery hospitalizations may be underestimated when examined using delivery hospitalizations as denominator, additional comparative analyses were performed. Firstly, we examined the incidence of ICU admission for the antepartum and postpartum hospitalizations, using the number of delivery hospitalizations as denominator for each. These analyses were performed to compare our findings with the only prior study [7] that examined specifically these categories of pregnancy-associated hospitalizations and used only delivery hospitalizations as denominator. We then reanalyzed the incidence of ICU admission within antepartum and postpartum hospitalizations (i.e., using all postpartum hospitalizations as denominator for ICU admissions of postpartum hospitalizations).

Death among ICU admissions was examined as hospital mortality (defined as the number of pregnancy-associated ICU admissions who died in the hospital divided by the total number of pregnancy-associated ICU admissions).

We examined the temporal trends of ICU utilization among all ICU-managed pregnancy-associated hospitalizations using linear least squares regression of log-transformed data to derive an average annual percent change (AAPC) and corresponding 95\% confidence intervals (95\% CIs). Stratified analyses of the temporal trends of ICU utilization were then carried out for the subgroups of the primary outcome using 


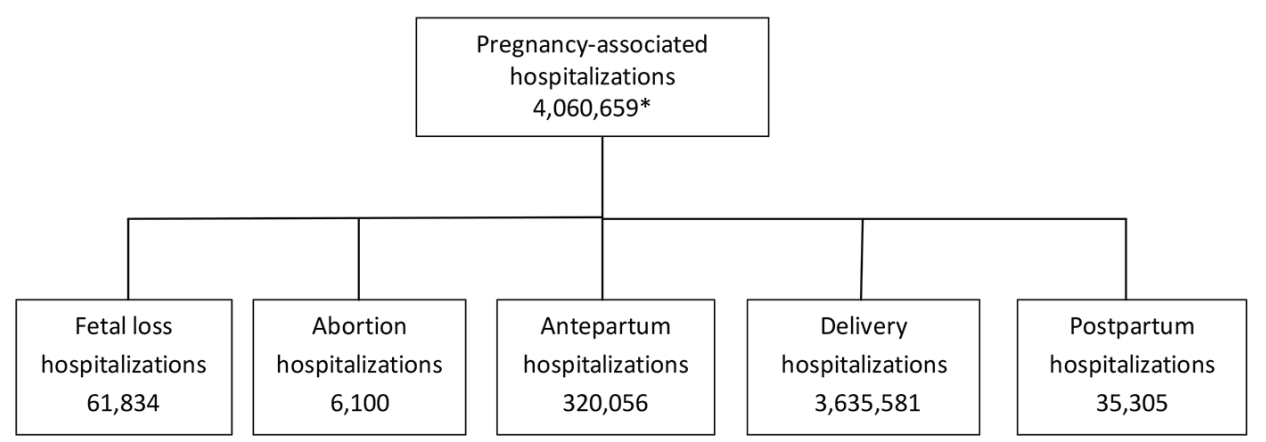

Figure 1. Categories of pregnancy-associated hospitalizations in Texas, $2001-2010$. *There were $1,781(0.04 \%)$ pregnancyassociated hospitalizations that could not be classified as either fetal loss or abortion.

similar approach.

Because temporal changes in ICU utilization may be affected by changes in threshold of ICU admission, we examined corresponding temporal trends in severity of illness and hospital mortality among ICU admissions. We thus converted the APR-DRG severity of illness qualitative categories described earlier to a quantitative four-category score ranging from 1 for "mild" thorough 4 for "extreme" for individual hospitalizations, similar to the approach described by Baram and colleagues [26]. We then calculated the corresponding mean annual severity of illness score to allow trend analysis, using the approach described above for trending ICU utilization.

Group data are reported as counts and percentages for categorical variables. All statistical analyses were performed using MedCalc version 15.6.1 (MedCalc Software, Ostend, Belgium) and SAS version 9.3 (SAS Institute, Cary, NC, USA). A two-sided $\mathrm{P}$ value $<0.05$ was considered statistically significant.

\section{Results}

The categories of pregnancy-associated hospitalizations are described in Figure 1. There were 158,410 pregnancy-associated hospitalizations involving ICU admission, with their characteristics detailed in Table 1 . Women aged $\geq 35$ years comprised $14.8 \%$ of the ICU cohort. Comorbid conditions were reported only in a minority $(9.7 \%)$, as was the occurrence of one or more organ dysfunctions (6.2\%). Chronic lung disease and diabetes were the most commonly reported comorbidities. Delivery hospitalizations (73.7\%) comprised the most common category of pregnancy-associated hospitalization among ICU admissions. There were 1,981 ICU admissions $(1.3 \%)$ with stillbirths. Severity of illness was reported as mild in $37.8 \%$, being extreme only in $3.6 \%$. Hospital mortality was rare among ICU admissions ( $0.3 \%)$, with the majority (97.6\%) discharged home.

\section{Incidence of ICU admission}

The overall incidence of ICU admission and that across cat- egories of pregnancy outcomes and pregnancy-associated hospitalizations are detailed in Table 2. The incidence of ICU admission was 29.6 per 1,000 TEP-years and 39.0 per 1,000 pregnancy-associated hospitalizations-years. There was marked variability in ICU utilization within specific categories of pregnancy outcomes, ranging from 0.6 per 1,000 abortionyears to 85.9 per 1,000 stillbirths-years. When the incidence of ICU admission was indexed to delivery hospitalizations, delivery hospitalizations had the highest ICU utilization at 32.1 per 1,000 delivery hospitalization-years, with the lowest utilization rate noted for postpartum hospitalizations, at 1.4 per 1,000 delivery hospitalizations-years.

However, once examined within each category of pregnancy-associated hospitalization, the findings reversed, with the incidence of ICU admission being highest among postpartum hospitalizations at 144.8 per 1,000 postpartum hospitalization-years.

\section{Temporal trends}

The temporal changes in the incidence of ICU admission are outlined in Table 2. The incidence of ICU admission rose among both TEP and all pregnancy-associated hospitalization by $63.9 \%$ and $68.0 \%$, respectively between 2001 and 2010 and increased variably in all examined subgroups, with the exception of abortion. ICU utilization dropped $47 \%$ among women who had abortion in the state, but remained unchanged among those who were hospitalized.

Severity of illness as measured by mean APR-DRG scores showed annual decrease of $-1.1 \% /$ year ( $95 \%$ CI: $-2.2 \%$ to $-0.06 \% ; \mathrm{P}=0.0413$ ) from 2.1 to 1.9 between 2001 and 2010 , respectively. Hospital mortality among ICU admissions remained unchanged (AAPC -1.1\%/year; $95 \% \mathrm{CI}:-3.9 \%$ to $+1.7 \% ; \mathrm{P}=0.4041)$.

\section{ICU utilization with specific maternal complications and organ dysfunction}

The occurrence of specific maternal complications and organ dysfunction among ICU admissions and the rate of ICU utilization for each among all pregnancy-associated hospitalization 
Table 1. The Characteristics of Pregnancy-Associated ICU Admissions

\begin{tabular}{|c|c|}
\hline Characteristic & $n=158,410$ \\
\hline \multicolumn{2}{|l|}{ Age $(\text { years, } n(\%))^{a, b}$} \\
\hline$<18$ & $7,481(4.8)$ \\
\hline $18-34$ & $124,898(80.4)$ \\
\hline$\geq 35$ & $22,994(14.8)$ \\
\hline \multicolumn{2}{|l|}{ Race/ethnicity, n (\%) } \\
\hline Hispanic & $53,491(33.8)$ \\
\hline White & $60,525(38.2)$ \\
\hline Black & $30,027(19.0)$ \\
\hline Other & $14,327(9.0)$ \\
\hline Missing & $40(<0.1)$ \\
\hline \multicolumn{2}{|l|}{ Health insurance, $\mathrm{n}(\%)$} \\
\hline Private & $73,075(46.10)$ \\
\hline Medicaid & $71,179(45.0)$ \\
\hline Uninsured & $10,465(6.6)$ \\
\hline Other & $3,619(2.3)$ \\
\hline Missing & $72(<0.1)$ \\
\hline \multicolumn{2}{|l|}{ Select comorbidities, $\mathrm{n}(\%)^{\mathrm{c}}$} \\
\hline Any & $15,334(9.7)$ \\
\hline Lung disease & $5,133(3.2)$ \\
\hline Connective tissue disease & $836(0.5)$ \\
\hline Liver disease & $735(0.4)$ \\
\hline Diabetes & $4,625(2.9)$ \\
\hline Renal disease & $628(0.3)$ \\
\hline Malignancy & $321(0.2)$ \\
\hline HIV & $120(0.1)$ \\
\hline \multicolumn{2}{|c|}{ Categories of pregnancy-associated hospitalizations, n (\%) } \\
\hline Delivery & $116,691(73.7)$ \\
\hline Antepartum & $31,751(20.0)$ \\
\hline Postpartum & $5,244(3.3)$ \\
\hline Fetal loss ${ }^{\mathrm{d}}$ & $3,009(1.9)$ \\
\hline Abortion $^{\mathrm{d}}$ & $449(0.3)$ \\
\hline \multicolumn{2}{|l|}{ Organ dysfunction, $\mathrm{n}(\%)$} \\
\hline Any & $9,769(6.2)$ \\
\hline$\geq 3$ & $449(0.3)$ \\
\hline \multicolumn{2}{|l|}{ APR-DRG severity of illness, n (\%) } \\
\hline Mild & $59,951(37.8)$ \\
\hline Moderate & $56,373(35.6)$ \\
\hline High & $36,312(22.9)$ \\
\hline Extreme & $5,774(3.6)$ \\
\hline \multicolumn{2}{|l|}{ Hospital disposition, $\mathrm{n}(\%)$} \\
\hline Death & $414(0.3)$ \\
\hline Hospice & $24(<0.1)$ \\
\hline Another facility & $2,213(1.4)$ \\
\hline Home & $154,656(97.6)$ \\
\hline Leave against medical advice & $1,103(0.7)$ \\
\hline
\end{tabular}

aPercent figures are rounded. ${ }^{b}$ Age groups were reported as $<17,18-44$, and $\geq 45$ for 3,037 (1.9\%) of ICU admissions with a diagnosis of human immunodeficiency virus infection, drug or alcohol abuse as additional means to preserve confidentiality in the data set. The denominator used to derive age group frequency for the cohort was based on hospitalizations with available 5-year age group designation $(n=155,373)$. "Based on comorbidities included in the Deyo-Charlson index. ${ }^{\mathrm{d}}$ The figures for fetal loss and abortion do not include ICU $1,266(0.8 \%)$ hospitalizations for which it could not be determined whether they involved fetal loss or abortion. HIV: human immunodeficiency virus. 
Table 2. The Incidence of Pregnancy-Associated ICU Admissions

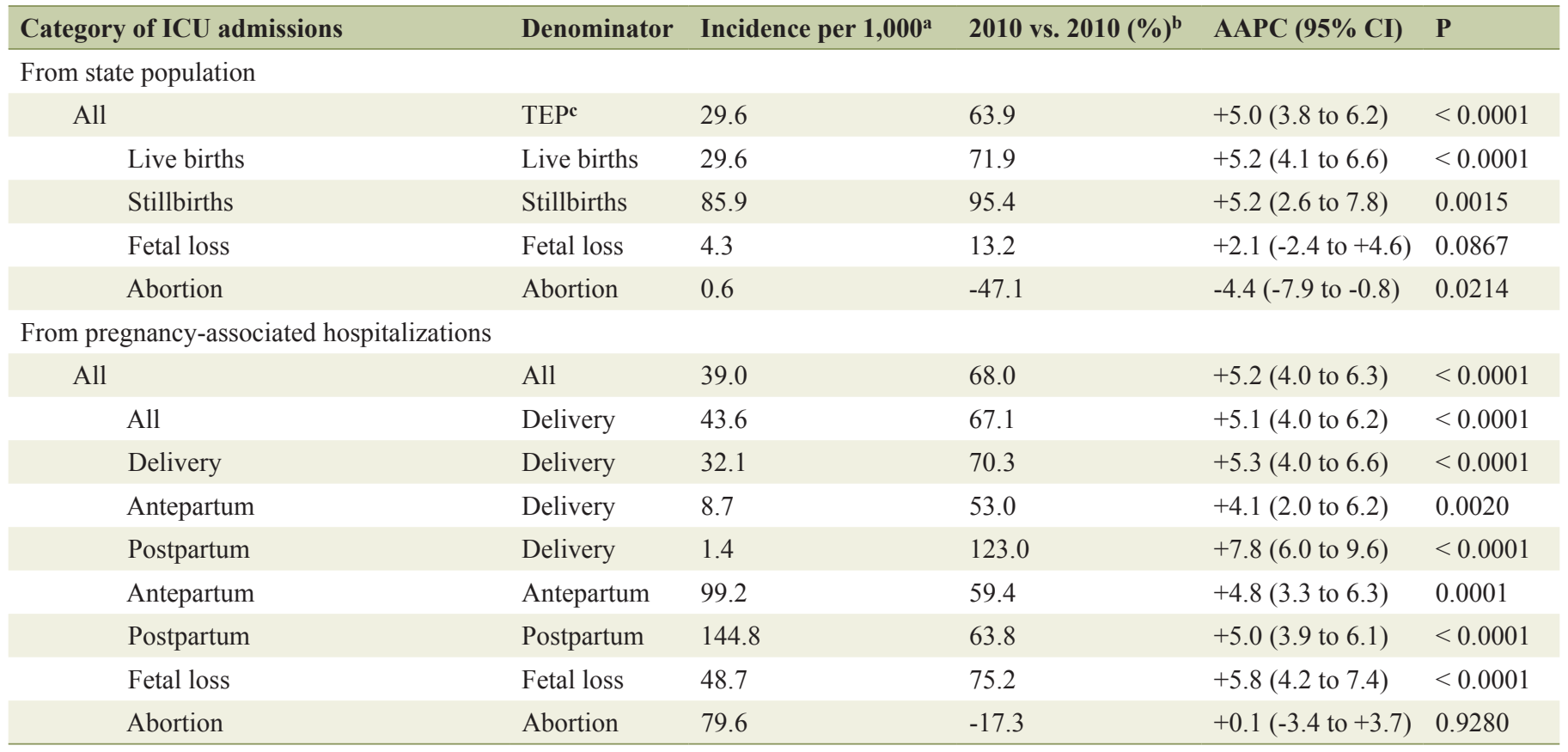

ancidence estimates are per 1,000 of a given denominator-years (i.e., per 1,000 live births-years). ${ }^{b}$ Refers to the relative difference. AAPC: average annual percent change; $95 \% \mathrm{Cl}: 95 \%$ confidence intervals. ${ }^{\mathrm{C} T E P}$ : total estimated pregnancies.

with the examined conditions are described in Table 3.

Preeclampsia/eclampsia $(23.3 \%)$ and obstetric hemorrhage $(6.9 \%)$ were the most common maternal complications among ICU admissions. However, only a minority of pregnancy-associated hospitalizations with each were admitted to ICU, at rates of $12.0 \%$ and $8.9 \%$, respectively. On the other hand, nearly $66 \%$ of those with status epilepticus received care in the ICU, though comprising less than $0.1 \%$ of ICU admissions. Similarly, marked variability was noted between frequency of specific maternal complications among ICU admissions and the corresponding use of ICU among all hospitalizations with specific conditions.

Organ dysfunction affected most commonly the respiratory and hematological systems among ICU admissions and high rates of ICU admission were generally observed among hospitalizations with each organ dysfunction, being the highest among those respiratory and hepatic dysfunction.

\section{Discussion}

In this population-based analysis of pregnancy-associated hospitalizations in Texas, ICU utilization was common. There has been high variability in ICU utilization across specific subgroups of pregnancy outcomes, being highest among women having stillbirths. Marked heterogeneity in ICU utilization was also noted within specific categories of pregnancy-associated hospitalization, being lowest during delivery hospitalizations and highest during postpartum hospitalization. ICU utilization rose considerably during the past decade for the whole cohort and nearly all examined subgroups, while severity of illness decreased modestly. Preeclampsia/eclampsia and obstetric hemorrhage were the most common maternal complications among ICU-managed hospitalizations, though the majority of hospitalizations with these conditions were managed outside ICU. Hospital mortality was rare and the vast majority of ICU admissions were discharged home.

\section{Incidence of ICU admissions}

We found that nearly 1 in 25 pregnancy-associated hospitalizations in Texas involved admission to ICU, the highest reported population-based rate in the US and nearly 26 -fold higher than the lowest reported figure [15]. Previously reported incidence of ICU admission in state-level studies ranged from 1.5 (during 1984 - 1997 [15]) to 4.2 (during 1998 - 2008 [7]) per 1,000 deliveries in Maryland and 15.4 (during 1997 - 2005) per 1,000 deliveries in New Jersey [16].

The contributions of variation in ICU bed availability, patient case-mix, and health care system organization and practice patterns have to be considered in the interpretation of remarkable across-state variability in demand for ICU services in the US. The US has among the highest population-indexed number of adult ICU beds in high-resource countries [17], but there have not been, to our knowledge, similar state-specific data. Increasing number of ICU beds was suggested as a key driver of rising ICU utilization in the general population [27]. However, while some variability can be expected across sates in population-adjusted ICU bed numbers, it is unlikely that the variability is of such magnitude as to the explanation the aforementioned reported difference in ICU utilization. Moreover, 
Table 3. Occurrence of Maternal Complications and Organ Dysfunctions Among ICU Admissions $(n=158,410)$ and Corresponding Rates of ICU Admission Among Pregnancy-Associated Hospitalizations

\begin{tabular}{|lll}
\hline Category & Occurrence among ICU admissions (n (\%)) & Rate of ICU admission (\%) \\
\hline Maternal complications & & \\
\hline Preeclampsia/eclampsia & $36,978(23.3)$ & 12.0 \\
\hline Hemorrhage & $11,005(6.9)$ & 8.9 \\
\hline Embolism & $1,020(0.6)$ & 51.0 \\
\hline Cardiovascular conditions & $9,603(6.1)$ & 15.2 \\
\hline Cardiomyopathy & $2,740(1.7)$ & 63.5 \\
\hline Cerebrovascular accident & $1,049(0.7)$ & 48.0 \\
\hline Sepsis & $1,746(1.1)$ & 51.3 \\
\hline Aspiration pneumonia & $583(0.4)$ & 40.3 \\
\hline Trauma & $1,273(0.8)$ & 15.5 \\
\hline Anesthesia complications & $139(0.1)$ & 22.6 \\
\hline Hyperemesis & $2,120(1.3)$ & 5.0 \\
\hline Status asthmaticus & $154(0.1)$ & 25.0 \\
\hline Status epilepticus & $80(<0.1)$ & 65.6 \\
\hline Blood transfusion complication & $68(<0.1)$ & 22.2 \\
\hline Organ dysfunction & & \\
\hline Respiratory & $4,470(2.8)$ & 73.5 \\
\hline Cardiovascular & $1,815(1.1)$ & 40.3 \\
\hline Renal & 1,051 & 64.9 \\
\hline Hepatic & $203(0.1)$ & 70.2 \\
\hline Hematological & $3,933(2.5)$ & 15.6 \\
\hline Metabolic & $968(0.6)$ & 58.4 \\
\hline Neurological & $572(0.4)$ & 61.1 \\
\hline
\end{tabular}

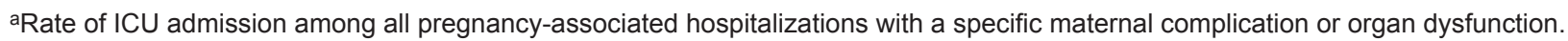

even when availability of ICU beds is nearly similar, as is the case in the US and Belgium, the incidence of ICU admission was nearly double in the former [17].

The frequency of severe maternal morbidity can be expected to vary across states in the US, subject among other factors to regional variability in demographics and access to healthcare. Although variability in maternal morbidity likely contributed to the observed heterogeneity in ICU utilization rates, it does not appear plausible that the former would differ nearly four-fold between two contingent US states [7, 16]. Overall women's severity of illness may have varied between states. However, severity of illness was not reported in prior US-based population studies, precluding more direct comparison when inferring sources of variability of ICU utilization patterns. Of note, there was markedly higher frequency of maternal complications among ICU admissions in Maryland [7] than in Texas. The latter differences support the hypothesis that the markedly higher incidence of ICU admission in Texas was not driven predominantly by higher burden of maternal morbidity. It can thus be postulated that between-state variability in clinical practice and health care system organizational factors have been the major drivers of the tremendous variability in the observed ICU utilization among obstetric patients, with likely markedly lower threshold for ICU admission in Texas.

Only one population-based study examined ICU utilization during antepartum and postpartum hospitalizations [7]. However, although indexing ICU utilization to delivery hospitalizations can provide a readily available denominator, the results reflect the predominance of delivery hospitalizations (comprising over $90 \%$ of all pregnancy-associated hospitalizations in the latter study [7]). This approach led, as was demonstrated in our study, to substantial underestimation of the actual relative demand for critical care resources within individual categories of pregnancy-associated hospitalizations. We found that once stratified within specific categories of pregnancy-associated hospitalizations, ICU utilization among antepartum and postpartum hospitalizations exceeded that of delivery hospitalizations by nearly three- and five-fold, respectively. Although Wanderer et al did not provide separately the number of all antepartum and postpartum hospitalizations in their population, the calculated incidence of ICU admission was 22.4 per 1,000 non-delivery hospitalizations [7], which is 10-fold higher than that among delivery hospi- 
talizations in their cohort. Our findings that women hospitalized during postpartum period have the highest incidence of ICU admission among pregnancy-associated hospitalizations extend earlier data showing that, as compared with delivery hospitalizations, the former group has 14-fold higher rates of severe maternal morbidity [28] and 32-fold higher rate of hospital mortality [29].

We estimated for the first time ICU utilization in the obstetric population, both overall and across specific pregnancy outcomes, showing that on average 1 in 30 pregnancies in the state involved ICU admission. Prior studies reported estimates of ICU utilization among all pregnant women, based exclusively on pregnancy-associated hospitalizations. This approach excludes, however, key subgroups that are not hospitalized (i.e., fetal loss, abortion). Thus, our study provides broader perspective of the demand for ICU services for the obstetric population.

The incidence of ICU admission varied 143-fold across different pregnancy outcomes, ranging from 1 in 1,700 for women having an abortion to nearly 1 in 12 among women with stillbirths. When considered from a population perspective, these findings reflect the remarkable safety of contemporary abortion. On the other hand, the factors underlying the extremely high demand for critical care resources among women having stillbirths is less clear. Stillbirths are considered to represent a relatively neglected area in the global initiative to improve maternal and fetal outcomes [30]. Only limited data are available on maternal conditions associated with stillbirths in high-resource countries [30], while a recent audit of stillbirths in South Africa demonstrated associated maternal conditions in the majority of women, with the most common representing those associated with high maternal morbidity [31]. Nevertheless, our findings suggest that women with stillbirths represent a remarkably high-risk obstetric group from maternal perspective.

Hospitalized women with fetal loss or abortion had, as expected, markedly higher incidence of ICU admission than those with delivery hospitalizations, extending the findings reported by Wanderer et al, when the investigators combined both groups [7]. This is because, similarly to antepartum and postpartum hospitalizations, the former groups represent admissions for complications. However, once hospitalized, the need for ICU care was much higher among those women with abortion than those with fetal loss, suggesting higher burden of morbidity among the former.

\section{Temporal trends in ICU utilization}

Our findings of progressive rise in the incidence of ICU admission in Texas contrast those of unchanged ICU utilization among obstetric hospitalizations in Maryland, reported by Wanderer et al [7]. The rate of severe maternal morbidity in the US increased by $107 \%$ between 2000 - 2001 and 2010 - 2011 [4] and would be expected to impact ICU utilization over time, even if to a different magnitude, within all states. However, the sources of the conflicting findings are unclear and the results are of special note since ICU utilization by obstetric patients in Maryland rose 69\% between 1984 - 1997 [15] and 1999 and 2008 [7], even when considering only delivery hospitali- zations. The investigators of the latter study speculated that the unchanged ICU utilization rates in Maryland may represent change in threshold of ICU admission, coding practices, or that trends in maternal morbidity in the state do not correlate with national trends [7].

We found decreasing annual severity of illness of ICU admissions. This change may support a possibility of decreasing threshold of ICU admission over time. However, while statistically significant, the annual absolute and relative changes were clinically trivial and unlikely to be the major drivers of the observed rapid rise in ICU utilization in Texas. The lack of severity of illness data in the study by Wanderer and colleagues [7] limits further comparisons. Additional studies of the temporal patterns of ICU utilization and patients' severity of illness measures coupled with corresponding examination of broader characteristics of state-specific obstetric population can provide further insight into the conflicting findings and inform resource allocation.

\section{ICU utilization with specific maternal complications and organ dysfunction}

Preeclampsia/eclampsia and obstetric hemorrhage were the most common reported maternal complications among ICU admissions in Texas, similar to other reports in the US [6-8] and a commonly used summary metric of ICU admissions in this population $[6,8]$. However, this basic summary measure obscures more complex variability in practice approach to ICU utilization in obstetric patients.

When comparing rates of ICU use for the examined maternal complications and organ dysfunction between Texas and Maryland (the only other study providing, though indirectly, rates of ICU use) [7], we found nearly uniformly markedly higher rates of admission to ICU for each of the examined complications and organ dysfunctions, as compared to those described by Wanderer and colleagues. It may be hypothesized that the severity of individual maternal complications and organ dysfunctions was substantially higher in the Texas population. However, the frequency of each among ICU admissions was markedly lower than that reported by others [7], consisting only a minority of all ICU admissions in the state and with the overall maternal mortality among ICU admissions markedly lower than that noted in the Maryland cohort [7]. The latter findings support the likely lower threshold for ICU admission of obstetric patients by Texas clinicians.

We found that high or extreme severity of illness occurred only in about one in four of ICU admissions in our study. Our findings cannot be compared to other US-based populationbased studies, because none reported severity of illness measures. However, when physiological measures of severity of illness of ICU managed obstetric patients were examined in other population-based studies in high-resource countries with more restrictive admission patterns, the investigators found that only a minority had organ dysfunction, with even smaller fraction requiring life-support interventions [10]. The latter data support a hypothesis that ICU resources in Texas were commonly used, well beyond the aforementioned traditional 
high-risk obstetric patient groups, for closer patient monitoring, rather than interventional purpose.

\section{ICU utilization and maternal outcomes}

The rate of maternal death in our study was toward the lower end of reported population-level data $[7,15,16]$. There has been an inverse relationship between the incidence of ICU admission and maternal death, with 26-fold difference in the incidence of ICU admission and 22-fold difference in hospital mortality across US studies $[15,16]$. However, the rare occurrence of maternal death limits inference about the overall impact of varying reported ICU utilization.

The majority $(58.6 \%)$ of maternal deaths in the study by Wanderer et al occurred without ICU admission [7]. The investigators indicated that though the threshold for ICU utilization in Maryland may be lower than elsewhere, the demonstrated plurality of maternal deaths occurring without ICU admission could suggest that ICU was still underutilized in their population. We recently reported that $26 \%$ of deaths occurring in pregnancy-associated hospitalizations in Texas were not preceded by admission to ICU [29]. However, the markedly lower rate of maternal death occurring outside the ICU in Texas does not necessarily confirm more appropriate ICU utilization in the state. Indeed, occurrence of maternal death exclusively in the ICU setting does not equate timely and appropriate triage and care (see below).

Prior reports on maternal deaths in the US showed high rates of preventable morality among decedents, related predominantly to physician and health system factors, stemming from inadequate early recognition of maternal crises and delayed escalation of care, including transfer to $\operatorname{ICU}[32,33]$. The administrative data used in our study and in prior population investigations in the US $[7,15,16]$ do not provide information on the timeliness of problem recognition by clinicians, timeliness of ICU admission, and appropriateness of care around and following ICU transfer, both among women who died, as well as the remainder of ICU-managed patients. Nor can these large data sets inform investigators about the association between rates of ICU utilization and potentially preventable maternal death or additional maternal morbidity. The latter measures of patient-centered benefit of ICU-based care cannot be ethically examined in a randomized experimental approach. Rather, the demonstrated tremendous practice variability in ICU utilization among obstetric patients, and substantial difference in demand for ICU within specific groups related to pregnancy outcomes and type of hospitalization make a compelling case for future observational studies with sufficient granularity of data to inform clinicians, health care systems and policy-level decisions.

Because maternal mortality is a relatively rare event among ICU-managed obstetric patients in high-resource countries, outcomes of survivors can provide broader perspective when comparing study findings on benefits of ICU-based care. We found that the vast majority of ICU-managed pregnancyassociated hospitalizations in Texas were discharged home. The later findings indicate a remarkably low short-term residual morbidity at the time of hospital discharge. However, only mortality data were reported by other investigators in population-based studies, precluding further comparisons or inference about the benefits of the varying ICU utilization patterns.

\section{Implications of the present study}

Our findings confirm that patterns of ICU utilization are an inappropriate proxy for study of the burden of severe maternal morbidity or near-miss events, at least in a high-resource health care environment, with high supply of ICU beds.

The threshold for ICU admission may be lower in obstetric patients than among their non-obstetric counterparts with otherwise similar severity of illness, when fetal and neonatal outcomes are considered. However, prior studies consistently demonstrated that only a minority of obstetric patients (median $16 \%$ ) were still pregnant at the time of ICU admission [6]. Thus, ICU admission considerations would be expected to be comparable between the non-pregnant majority of obstetric patients and non-obstetric population. However, our findings suggest that at a population level clinicians may be using markedly more lenient criteria when considering ICU admission in the former.

Given the noted variability in ICU utilization across states in the US, specific ICU utilization rates for each of the subgroups examined in the present study are likely to differ considerably in other healthcare settings and in different populations. However, our findings demonstrate for the first time the substantial clinically relevant heterogeneity in demand for critical care resources within specific pregnancy outcomes and pregnancy-associated hospitalization groups. These latter data identify, in turn, specific high-risk populations, requiring heightened vigilance for early recognition and timely triage and intervention. In addition, our findings may complement the current approach based on severe maternal morbidities and near-miss events, both as targets for future study of preventive and interventional strategies and as potential performance improvement measure within and across national boundaries. Finally, these stratified data can help refine comparative study of resource utilization across populations and healthcare systems and inform clinicians, investigators and health care policy makers.

While timely and effective care in an ICU can be lifesaving, ICU admission has a well-known downside, ranging from risk of ICU-acquired infections with multidrug-resistant pathogens [34] and delirium [35], to patient and family anxiety [36], with associated short-term [37] and long-term [38] adverse impact. In addition, unnecessary ICU admission can limit timely access for sicker patients, which can lead to higher risk of death [39]. Thus, our study adds to the accumulating data underscoring the compelling case for measures to identify and effectively address sources of variability in ICU utilization among obstetric patients in high-resource care environments.

\section{Strengths and limitations}

The key strengths of the present study include use of a highquality data set in a state with diverse population, representing 
the largest cohort reported to date of ICU-managed obstetric patients. The studied population thus allowed for robust estimates of the epidemiology of ICU utilization, with the findings transcending inter-facility variability.

The study has several limitations due to the retrospective study design and use of administrative data. In addition to the limitations noted earlier, dependence on ICD-9 based coding may have resulted in misclassification of some pregnancyassociated hospitalizations, maternal complications and organ dysfunction. However, pregnancy-associated hospitalizations were the most common category of hospitalization [40] in Texas, with expected high clinician and coder familiarity. Thus, a systematic bias in the annual reporting or general underestimation of pregnancy-associated hospitalizations is unlikely. Our approach to identify maternal morbidity and organ dysfunction was similar to that used by investigators [24, 28, 41].

In addition, although our study provided a broad overview of state-level practice patterns of ICU utilization in obstetric patients, the structure of the data set precluded insight into health system organizational and practice factors that are bound to affect triage to ICU. Finally, as the present study expanded the growing data on the tremendous across-state variability in ICU utilization among obstetric patients in the US, our findings are not generalizable to other states. However, the noted heterogeneity in ICU utilization across the examined subgroups of obstetric patients may be considered as benchmark for further studies of the relative demand for critical care resources in other populations and healthcare settings.

\section{Conclusion}

We found that pregnancy-associated ICU utilization in Texas was the highest reported in the US, rising progressively over the past decade. Tremendous heterogeneity in demand for critical care resources was demonstrated across pregnancy outcomes and categories of pregnancy-associated hospitalizations. The latter findings identify for the first time broader highrisk patient groups requiring closer vigilance and timely triage and care. At the same time, our findings suggest markedly low threshold for ICU admission of obstetric patients in the state, as evidenced by comparatively rare maternal death and likely minimal, if any, residual morbidity among hospital survivors. These findings underscore the need to limit avoidable practice variability in ICU utilization in the obstetric population in the US to both enhance maternal, fetal and neonatal outcomes and to optimize limited resource allocation.

\section{Acknowledgments}

I would like to thank Phillip Watkins, MS, for his help with parts of data acquisition and management.

\section{Conflicts of Interests}

The author declares that there are no conflicts of interest re- garding the publication of this paper.

\section{Grant Support}

None.

\section{References}

1. Martin JA, Hamilton BE, Ventura SJ, et al. Births: First data for 2010. National vital statistics reports; vol 61 no1. Hyattsville, MD: National Center for Health Statistics. 2012. Available at: http://www.cdc.gov/nchs/data/NVSR/ NVSR61/NVSR61_01.pdf.

2. Campbell KH, Savitz D, Werner EF, Pettker CM, Goffman D, Chazotte C, Lipkind HS. Maternal morbidity and risk of death at delivery hospitalization. Obstet Gynecol. 2013;122(3):627-633.

3. Barber EL, Lundsberg LS, Belanger K, Pettker CM, Funai $\mathrm{EF}$, Illuzzi JL. Indications contributing to the increasing cesarean delivery rate. Obstet Gynecol. 2011;118(1):2938 .

4. Severe maternal morbidity in the United States. Reproductive Health. Centers for Disease Control and Prevention, 2016. Available at: http://www.cdc.gov/reproductivehealth/maternalinfanthealth/severematernalmorbidity.html.

5. Creanga AA, Berg CJ, Syverson C, Seed K, Bruce FC, Callaghan WM. Pregnancy-related mortality in the United States, 2006-2010. Obstet Gynecol. 2015;125(1):5-12.

6. Pollock W, Rose L, Dennis CL. Pregnant and postpartum admissions to the intensive care unit: a systematic review. Intensive Care Med. 2010;36(9):1465-1474.

7. Wanderer JP, Leffert LR, Mhyre JM, Kuklina EV, Callaghan WM, Bateman BT. Epidemiology of obstetric-related ICU admissions in Maryland: 1999-2008*. Crit Care Med. 2013;41(8):1844-1852.

8. Zeeman GG. Obstetric critical care: a blueprint for improved outcomes. Crit Care Med. 2006;34(9 Suppl):S208-214.

9. Say L, Souza JP, Pattinson RC. Maternal near miss - towards a standard tool for monitoring quality of maternal health care. Best Pract Res Clin Obstet Gynaecol. 2009;23(3):287-296.

10. Chantry AA, Deneux-Tharaux C, Bonnet MP, BouvierColle MH. Pregnancy-related ICU admissions in France: trends in rate and severity, 2006-2009. Crit Care Med. 2015;43(1):78-86.

11. Zieleskiewicz L, Chantry A, Duclos G, Bourgoin A, Mignon $\mathrm{A}$, Deneux-Tharaux $\mathrm{C}$, Leone $\mathrm{M}$. Intensive care and pregnancy: Epidemiology and general principles of management of obstetrics ICU patients during pregnancy. Anaesth Crit Care Pain Med. 2016;35(Suppl 1):S51-S57.

12. Practice Bulletin No. 170: Critical Care in Pregnancy. Obstet Gynecol. 2016;128(4):e147-154.

13. Van Parys AS, Verstraelen H, Roelens K, Temmerman M. Maternal Intensive Care': a systematic literature review. Facts Views Vis Obgyn. 2010;2(3):161-167. 
14. Gaffney A. Critical care in pregnancy - is it different? Semin Perinatol. 2014;38(6):329-340.

15. Panchal S, Arria AM, Harris AP. Intensive care utilization during hospital admission for delivery: prevalence, risk factors, and outcomes in a statewide population. Anesthesiology. 2000;92(6):1537-1544.

16. Madan I, Puri I, Jain NJ, Grotegut C, Nelson D, Dandolu V. Characteristics of obstetric intensive care unit admissions in New Jersey. J Matern Fetal Neonatal Med. 2009;22(9):785-790.

17. Wunsch H, Angus DC, Harrison DA, Collange O, Fowler $\mathrm{R}$, Hoste EA, de Keizer NF, et al. Variation in critical care services across North America and Western Europe. Crit Care Med. 2008;36(10):2787-2793, e2781-2789.

18. Texas inpatient public use data file. Texas Health Care Information Center for Health Statistics. Texas department of state health services. Available at: http://www.dshs. state.tx.us/thcic/hospitals/Inpatientpudf.shtm.

19. Oud L, Watkins P. Contemporary trends of the epidemiology, clinical characteristics, and resource utilization of necrotizing fasciitis in Texas: a population-based cohort study. Crit Care Res Pract. 2015;2015:618067.

20. Kowaleski J. State definitions and reporting requirements for live births, fetal deaths, and induced termination of pregnancy (1997 revision). Hyattsville, Maryland: national Center for Health Statistics. 1997.

21. Vital statistics annual reports. Texas department of state health services. Available at: https://www.dshs.state. tx.us/chs/vstat/annrpts.shtm.

22. Kuklina EV, Whiteman MK, Hillis SD, Jamieson DJ, Meikle SF, Posner SF, Marchbanks PA. An enhanced method for identifying obstetric deliveries: implications for estimating maternal morbidity. Matern Child Health J. 2008;12(4):469-477.

23. Deyo RA, Cherkin DC, Ciol MA. Adapting a clinical comorbidity index for use with ICD-9-CM administrative databases. J Clin Epidemiol. 1992;45(6):613-619.

24. Lagu T, Rothberg MB, Shieh MS, Pekow PS, Steingrub JS, Lindenauer PK. Hospitalizations, costs, and outcomes of severe sepsis in the United States 2003 to 2007. Crit Care Med. 2012;40(3):754-761.

25. Oud L, Watkins P. Evolving trends in the epidemiology, resource utilization, and outcomes of pregnancy-associated severe sepsis: a population-based cohort study. J Clin Med Res. 2015;7(6):400-416.

26. Baram D, Daroowalla F, Garcia R, Zhang G, Chen JJ, Healy E, Riaz SA, et al. Use of the All Patient RefinedDiagnosis Related Group (APR-DRG) Risk of Mortality Score as a Severity Adjustor in the Medical ICU. Clin Med Circ Respirat Pulm Med. 2008;2:19-25.

27. Gooch RA, Kahn JM. ICU bed supply, utilization, and health care spending: an example of demand elasticity. JAMA. 2014;311(6):567-568.
28. Callaghan WM, Creanga AA, Kuklina EV. Severe maternal morbidity among delivery and postpartum hospitalizations in the United States. Obstet Gynecol. 2012;120(5):1029-1036.

29. Oud L. Patterns of the Demographics, Clinical Characteristics, and Resource Utilization Among Maternal Decedents in Texas, 2001 - 2010: A Population-Based Cohort Study. J Clin Med Res. 2015;7(12):937-946.

30. Lawn JE, Blencowe H, Pattinson R, Cousens S, Kumar R, Ibiebele I, Gardosi J, et al. Stillbirths: Where? When? Why? How to make the data count? Lancet. 2011;377(9775):1448-1463.

31. Saving babies 2008-2009: Seventh report on perinatal care in South Africa. Ed RC Pattinson. Tshepesa Press. Pretoria, 2011. Available at: http://www.ppip.co.za/wpcontent/uploads/Saving-Babies-2008-9.pdf.

32. Main EK, McCain CL, Morton CH, Holtby S, Lawton ES. Pregnancy-related mortality in California: causes, characteristics, and improvement opportunities. Obstet Gynecol. 2015;125(4):938-947.

33. Bauer ME, Lorenz RP, Bauer ST, Rao K, Anderson FW. Maternal Deaths Due to Sepsis in the State of Michigan, 1999-2006. Obstet Gynecol. 2015;126(4):747-752.

34. Brusselaers N, Vogelaers D, Blot S. The rising problem of antimicrobial resistance in the intensive care unit. Ann Intensive Care. 2011:1:47.

35. Brummel NE, Girard TD. Preventing delirium in the intensive care unit. Crit Care Clin. 2013;29(1):51-65.

36. Uhrenfeldt L, Aagaard H, Hall EO, Fegran L, Ludvigsen MS, Meyer G. A qualitative meta-synthesis of patients' experiences of intra- and inter-hospital transitions. J Adv Nurs. 2013;69(8):1678-1690.

37. Forster AJ, Kyeremanteng K, Hooper J, Shojania KG, van Walraven $C$. The impact of adverse events in the intensive care unit on hospital mortality and length of stay. BMC Health Serv Res. 2008;8:259.

38. Cuthbertson BH, Elders A, Hall S, Taylor J, MacLennan G, Mackirdy F, Mackenzie SJ. Mortality and quality of life in the five years after severe sepsis. Crit Care. 2013;17(2):R70.

39. Harris S, Singer M, Rowan K, Sanderson C. Delay to admission to critical care and mortality among deteriorating ward patients in UK hospitals: a multicentre, prospective, observational cohort study. Lancet. 2015;385 (Suppl 1):S40.

40. Facts and figures: statistics on hospital-based care in Texas, 2009. Texas Health Care Information Collection. DSHS Publication \# E87-11648. Available at: http:// www.dshs.state.tx.us/thcic/publications/hospitals/statisticalreports.shtm

41. Martin GS, Mannino DM, Eaton S, Moss M. The epidemiology of sepsis in the United States from 1979 through 2000. N Engl J Med. 2003;348(16):1546-1554. 